\title{
Graphite and Graphene as Perfect Spin Filters
}

\author{
V. M. Karpan, ${ }^{1}$ G. Giovannetti, ${ }^{1,2}$ P. A. Khomyakov, ${ }^{1}$ M. Talanana, ${ }^{1}$ A. A. Starikov, ${ }^{1}$ M. Zwierzycki, ${ }^{3}$ J. van den Brink, ${ }^{2,4}$ \\ G. Brocks, ${ }^{1}$ and P. J. Kelly ${ }^{1}$ \\ ${ }^{1}$ Faculty of Science and Technology and MESA ${ }^{+}$Institute for Nanotechnology, University of Twente, \\ P.O. Box 217, 7500 AE Enschede, The Netherlands \\ ${ }^{2}$ Instituut-Lorentz for Theoretical Physics, Universiteit Leiden, P. O. Box 9506, 2300 RA Leiden, The Netherlands \\ ${ }^{3}$ Institute of Molecular Physics, P.A.N., Smoluchowskiego 17, 60-179 Poznań, Poland \\ ${ }^{4}$ Institute for Molecules and Materials, Radboud Universiteit Nijmegen, P.O. Box 9010, 6500 GL Nijmegen, The Netherlands
}

(Received 7 June 2007; published 26 October 2007)

\begin{abstract}
Based upon the observations (i) that their in-plane lattice constants match almost perfectly and (ii) that their electronic structures overlap in reciprocal space for one spin direction only, we predict perfect spin filtering for interfaces between graphite and (111) fcc or (0001) hcp Ni or Co. The spin filtering is quite insensitive to roughness and disorder. The formation of a chemical bond between graphite and the open $d$-shell transition metals that might complicate or even prevent spin injection into a single graphene sheet can be simply prevented by dusting $\mathrm{Ni}$ or $\mathrm{Co}$ with one or a few monolayers of $\mathrm{Cu}$ while still preserving the ideal spin-injection property.
\end{abstract}

DOI: 10.1103/PhysRevLett.99.176602

The observation [1,2] of giant magnetoresistance in systems where the transmission through interfaces between normal and ferromagnetic metals (FM) is spin dependent has driven a major effort to study spin filtering effects in other systems and extend applications from field sensing to storage [3], reprogrammable logic [4], and quantum computing [5]. An ideal spin filter would allow all carriers with one spin through but none with the other spin. Interfaces with half-metallic ferromagnets (HMFs) [6] should have this property but progress in exploiting it has been slow because of the difficulty of making stoichiometric HMFs with the theoretically predicted bulk properties and then making devices maintaining these properties at interfaces [7].

If the nonmagnetic metal is replaced by an insulator (I) or semiconductor (SC), spin filtering still occurs giving rise to tunneling magnetoresistance (TMR) in FM|I|FM magnetic tunnel junctions and spin-injection at $\mathrm{FM} \mid \mathrm{SC}$ interfaces. If the spin polarization of the ferromagnet is not complete, then the conductivity mismatch between metals and semiconductors or insulators has been identified as a serious obstacle to efficient spin injection [8]. It can be overcome if there is a large spin-dependent interface resistance but this is very sensitive to the detailed atomic structure and chemical composition of the interface. Knowledge of the interface structure is a necessary preliminary to analyzing spin filtering theoretically and progress has been severely hampered by the difficulty of experimentally characterizing $\mathrm{FM} \mid \mathrm{I}$ and $\mathrm{FM} \mid \mathrm{SC}$ interfaces.

The situation improved with the confirmation of large values of TMR in tunnel barriers based upon crystalline $\mathrm{MgO}[9,10]$ which had been predicted by detailed electronic structure calculations [11,12]. While the record values of TMR - in excess of $500 \%$ at low temperatures [13] - are undoubtedly correlated with the crystallinity of
PACS numbers: 72.25. $-\mathrm{b}, 73.43 . \mathrm{Qt}, 75.47 .-\mathrm{m}, 81.05 . \mathrm{Uw}$

$\mathrm{MgO}$, the nature of this relationship is not trivial [14]. The sensitivity of TMR (and spin injection) to details of the interface structure $[15,16]$ make it difficult to close the quantitative gap between theory and experiment. In view of the reactivity of the open-shell transition metal (TM) ferromagnets $\mathrm{Fe}, \mathrm{Co}$, and $\mathrm{Ni}$ with typical semiconductors and insulators, preparing interfaces where disorder does not dominate the spin filtering properties remains a challenge. With this in mind, we wish to draw attention to a quite different material system which should be intrinsically ordered, for which an unambiguous theoretical prediction of perfect spin filtering can be made in the absence of disorder, and which is much less sensitive to interface roughness and alloy disorder than TMR or spin injection.

TABLE I. Lattice constants of $\mathrm{Co}, \mathrm{Ni}, \mathrm{Cu}$, and graphene. $a_{\text {hex }} \equiv a_{\text {fcc }} / \sqrt{2}$. Equilibrium separation $d_{0}$ for a layer of graphene on top of graphite, $\mathrm{Co}, \mathrm{Ni}$, or $\mathrm{Cu}$ calculated within the local density approximation (LDA) of density functional theory with $a=2.46 \AA$. The binding energy $\Delta E\left(d_{0}\right)=E(d=\infty)-$ $E\left(d_{0}\right)$ is the energy (per interface unit cell) required to remove a single graphene layer from a graphite stack or from a $\mathrm{Co}, \mathrm{Ni}$, or $\mathrm{Cu}$ (111) surface. $W$ is the work function.

\begin{tabular}{lclll}
\hline \hline & Graphene & \multicolumn{1}{c}{ Co } & \multicolumn{1}{c}{$\mathrm{Ni}$} & $\mathrm{Cu}$ \\
\hline$a_{\text {fcc }}^{\text {expt }}(\AA)$ & & $3.544^{\mathrm{a}}$ & $3.524^{\mathrm{a}}$ & $3.615^{\mathrm{a}}$ \\
$a_{\text {hex }}^{\text {expt }}(\AA)$ & 2.46 & 2.506 & 2.492 & 2.556 \\
$a_{\text {hex }}^{\mathrm{LDA}}(\AA)$ & 2.45 & 2.42 & 2.42 & 2.49 \\
$d_{0}(\AA)$ & 3.30 & 2.04 & 2.03 & 3.18 \\
$\Delta E\left(d_{0}\right)(\mathrm{eV})$ & 0.10 & 0.37 & 0.32 & 0.07 \\
$W_{\text {calc }}(\mathrm{eV})$ & 4.6 & 5.4 & 5.5 & 5.2 \\
$W_{\text {expt }}(\mathrm{eV})$ & $4.6^{\mathrm{b}}$ & $5.0^{\mathrm{c}}$ & $5.35^{\mathrm{c}}$ & $4.98^{\mathrm{c}}$ \\
\hline \hline
\end{tabular}

${ }^{\mathrm{a}}$ Ref. [17]

${ }^{\mathrm{b}}$ Ref. [18]

${ }^{\mathrm{c}}$ Ref. [19] 
We begin by observing that the in-plane lattice constants of graphene and graphite match the surface lattice constants of (111) $\mathrm{Co}, \mathrm{Ni}$, and $\mathrm{Cu}$ almost perfectly. From Table I, it can be seen that $\mathrm{Ni}$ is particularly suitable with a lattice mismatch of only $1.3 \%$. The second point to note is that the only electronic states at or close to the Fermi energy in graphene or graphite are to be found near to the high symmetry $\mathrm{K}$ point in reciprocal space where Co and $\mathrm{Ni}$ have states with minority spin character only. The absence of majority spin states in a large region about the $\mathrm{K}$ point is made clear in the (111) Fermi surface (FS) projections shown in Fig. 1. The (0001) FS projections for hcp Co are qualitatively the same. It follows that in the absence of symmetry-lowering (resulting from disorder, interface reconstruction, etc.) perfect spin filtering should occur for graphite on top of a flat Ni or Co (111) surface.

The effectiveness of the spin filtering is tested for a current-perpendicular-to-the-plane (CPP) structure with $n$ graphene layers sandwiched between semi-infinite Ni electrodes. The spin-dependent transmission through this $\mathrm{Ni}\left|\mathrm{Gr}_{n}\right| \mathrm{Ni}$ junction is calculated using a first-principles tight-binding muffin tin orbital (TB-MTO) wave-function

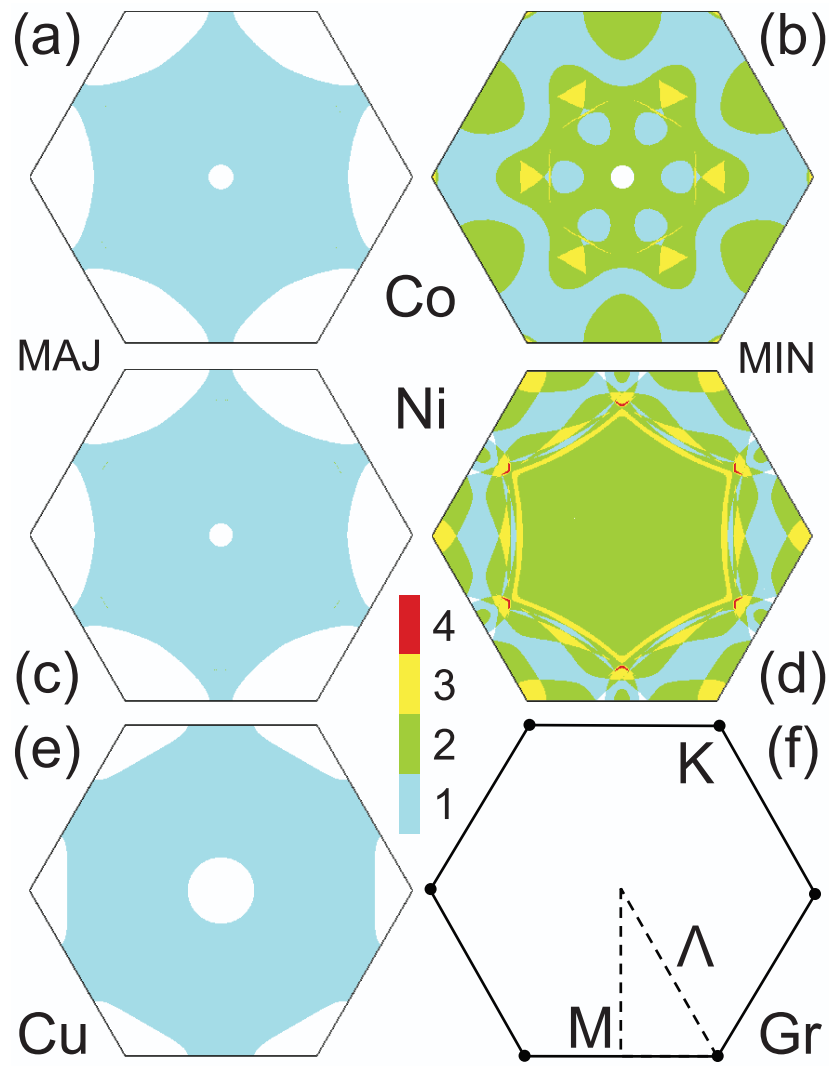

FIG. 1 (color). fcc FS projections onto a plane perpendicular to the [111] direction for Co majority (a) and minority (b) spins, for $\mathrm{Ni}$ majority (c) and minority (d) spins and for $\mathrm{Cu}$ (e). The number of FS sheets is shown by the color bar. For graphene and graphite, surfaces of constant energy are centered on the $\mathrm{K}$ point (f). matching scheme [20,21] for parallel (P) and antiparallel (AP) orientations of the Ni magnetizations. The atomic sphere (AS) potentials are calculated self-consistently within density functional theory for atomic structures determined by total energy minimization (see below). The conductances $G_{\mathrm{P}}^{\sigma}$ and $G_{\mathrm{AP}}^{\sigma}$ are shown in Fig. 2 for the minority and majority spin channels, $\sigma=\min$, maj. $G_{\mathrm{P}}^{\text {maj }}$ and $G_{\mathrm{AP}}^{\sigma}$ are strongly attenuated while, apart from an evenodd oscillation, $G_{\mathrm{P}}^{\min }$ is independent of $n$. The magnetoresistance $\mathrm{MR}=\left(R_{\mathrm{AP}}-R_{\mathrm{P}}\right) / R_{\mathrm{AP}} \equiv\left(G_{\mathrm{P}}-G_{\mathrm{AP}}\right) / G_{\mathrm{P}}$ rapidly approaches $100 \%$; see inset. We use the pessimistic definition of MR because $G_{\mathrm{AP}}$ vanishes for large $n$; usually the optimistic version is quoted [9-13]. Similar results are obtained for $\mathrm{Ni}\left|\mathrm{Gr}_{n}\right| \mathrm{Co}$ and $\mathrm{Co}\left|\mathrm{Gr}_{n}\right|$ Co junctions.

We envisage a procedure in which thin graphite layers are prepared by micromechanical cleavage of bulk graphite onto a $\mathrm{SiO}_{2}$ covered $\mathrm{Si}$ wafer [22] into which TM (Ni or $\mathrm{Co}$ ) electrodes have been embedded and layers of graphene are peeled away until the desired value of $n$ is reached. Assuming it will be possible to realize one essentially perfect interface in a CPP geometry, we studied the effect of roughness and disorder at the other interface on MR (inset Fig. 2). Replacing the top Ni layer with a $\mathrm{Ni}_{50} \mathrm{Cu}_{50}$ random alloy only reduces the MR to $90 \%$ (900\% in the optimistic definition). Extreme roughness, whereby half of

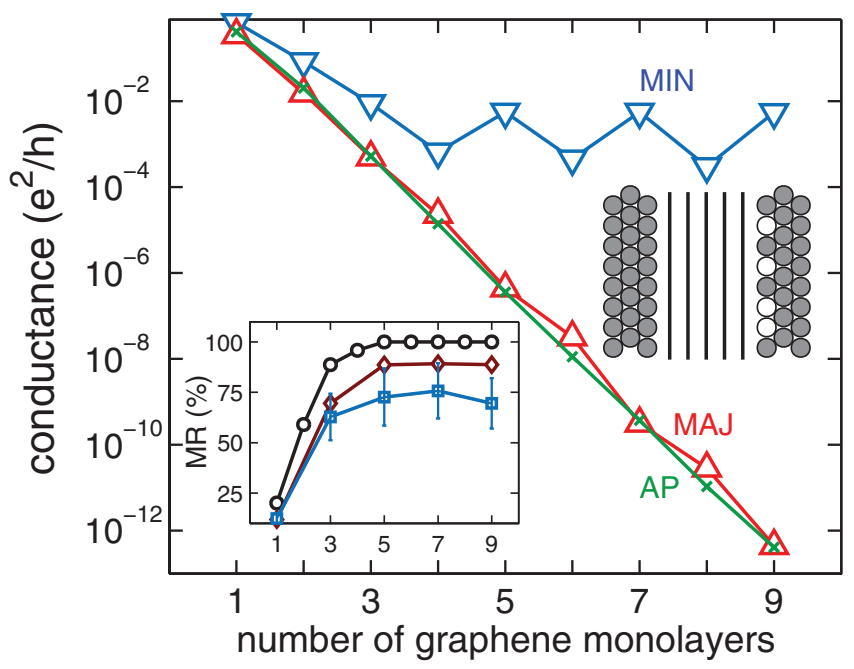

FIG. 2 (color). Conductances $G_{\mathrm{P}}^{\mathrm{min}}(\nabla), G_{\mathrm{P}}^{\mathrm{maj}}(\triangle)$, and $G_{\mathrm{AP}}^{\sigma}$ $(\times)$ of a $\mathrm{Ni}\left|\mathrm{Gr}_{n}\right| \mathrm{Ni}$ junction as a function of the number of graphene layers $n$ for ideal junctions. Inset: magnetoresistance as a function of $n$ for: (circles) ideal junctions; (diamonds) $\mathrm{Ni}\left|\mathrm{Gr}_{n}\right| \mathrm{Cu}_{50} \mathrm{Ni}_{50} \mid \mathrm{Ni}$ junctions where the surface layer is a disordered alloy; (squares) $\mathrm{Ni}\left|\mathrm{Gr}_{n}\right| \mathrm{Ni}$ junctions where the top layer of one of the electrodes is rough with only half of the top layer sites occupied (sketch). Results for roughness and disorder are modeled in $5 \times 5$ lateral supercells and averaged over 20 disorder configurations as described in Refs. [16,21]. For the rough surface layer, the error bars indicate the spread of MR obtained for different configurations. The supercell conductances are normalized to the $1 \times 1$ surface unit cell used for the ideal case. 
the $\mathrm{Ni}$ interface layer is removed at random, only reduces the MR to $70 \%$. The momentum transfer induced by the scattering is apparently insufficient to bridge the large gap about the $\mathrm{K}$ point in the majority spin FS projections. Alternatively, it may be possible to prepare two separate, near-perfect $\mathrm{TM} \mid \mathrm{Gr}$ interfaces and join them using a method analogous to vacuum bonding [23].

Graphite has a large $c$-axis resistivity [24]. If one of the $\mathrm{TM} \mid \mathrm{Gr}$ interfaces is ideal and the graphite layer is sufficiently thick, then it should be possible to achieve $100 \%$ spin accumulation in a high resistivity material making it suitable for injecting spins into semiconductors [8]. Because carbon is so light, spin-flip scattering arising from spin-orbit interaction should be negligible.

The results shown in Fig. 2 were calculated for the lowest energy "AC" configuration of graphene on $\mathrm{Ni}$ corresponding to one carbon atom above a $\mathrm{Ni}$ atom (the surface " $\mathrm{A}$ " sites) while the other is above a third layer Ni "C" site. A and C refer to the conventional ABC stacking of the layers in an fcc crystal (AB for an hcp structure). The CPP spin filtering should not depend on the details of how graphite bonds to the metal surface as long as the translational symmetry parallel to the interfaces is preserved. This is confirmed by explicit calculation for the "AB" and "BC" bonding configurations for varying graphene-metal surface separation $d$.

The nature of the bonding may well play an important role in realizing such an interface experimentally. In Fig. 3 we show the total energy of a graphene sheet on $\mathrm{TM}=\mathrm{Co}$, $\mathrm{Ni}$, and $\mathrm{Cu}(111)$ surfaces as a function of $d$ where the zero of energy has been chosen so that $E(d=\infty)=0$ for an uncharged graphene sheet. The density functional theory calculations were carried out using the projector augmented wave method [25,26], a plane wave basis set and the LDA, as implemented in the VASP program [27,28]. Supercells containing a slab of at least six layers of metal atoms with a graphene sheet adsorbed on one side of the

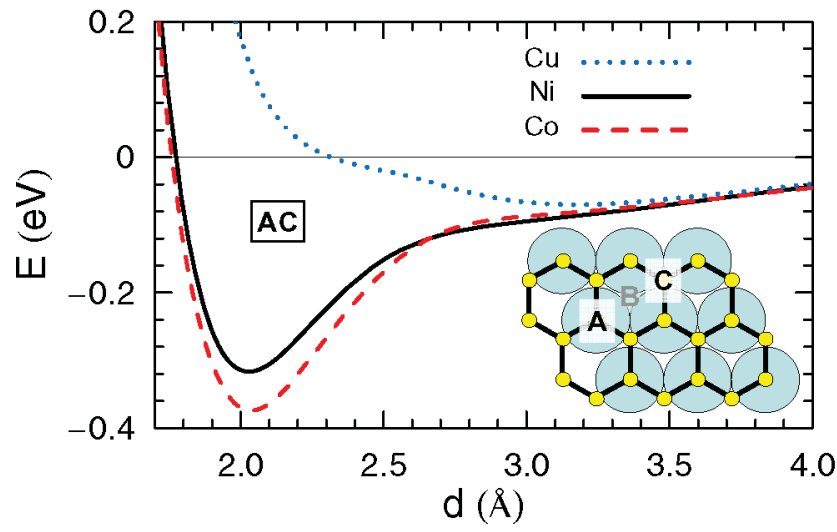

FIG. 3 (color). Total energy $E$ of a graphene sheet on fcc Co, $\mathrm{Ni}$, and $\mathrm{Cu}$ (111) surfaces as a function of the separation of the graphene sheet from the top layer of the metal. The lowest energy AC configuration is sketched on the bottom right. slab and a vacuum region of $\sim 12 \AA$ were used. The Brillouin zone of the $(1 \times 1)$ surface unit cell was sampled using a $36 \times 36 \mathbf{k}$-point grid. The plane wave kinetic energy cutoff was $400 \mathrm{eV}$. To avoid interactions between periodic images of the slab a dipole correction was applied [29]. The atoms in the metal layers were fixed at their bulk positions. The experimental lattice constant of graphene $a=2.46 \AA$ is used as the lattice parameter $a_{\mathrm{hex}}$ for $\mathrm{Co}, \mathrm{Ni}$ and $\mathrm{Cu}$. To plot the band structures (Fig. 4), a 13 layer slab with graphene absorbed on both sides was used.

The most prominent feature of Fig. 3 is the prediction of a weak minimum in the binding energy curve for $\mathrm{Cu}$ of about $0.07 \mathrm{eV}$ at an equilibrium separation $d \sim 3.2 \AA$ and deeper minima of 0.37 and $0.32 \mathrm{eV}$, respectively, for Co and $\mathrm{Ni}$, at a smaller equilibrium separation of $d \sim 2.0 \AA$. In agreement with a recent first-principles calculation [30] and experiment $[18,31]$ for graphene on $\mathrm{Ni}$, we find that the lowest energy corresponds to an AC configuration. The finer details of the total energy surfaces depend on the choice of exchange-correlation potential, relaxation of the metal substrate, choice of in-plane lattice constant, etc., and will be presented elsewhere. We restrict ourselves here to properties which do not depend on these details.

The electronic structure of a single graphene layer does depend on $d$. For the less strongly bound $\mathrm{BC}$ configuration of $\mathrm{Gr}$ on $\mathrm{Ni}$, the equilibrium separation is $d \sim 3.3 \AA$ and the characteristic band structure of an isolated graphene sheet is clearly recognizable; see Fig. 4 . For the lowest energy AC configuration, the interaction between the graphene sheet and Ni surface is much stronger, a gap is opened in the graphene derived $p_{z}$ bands and there are

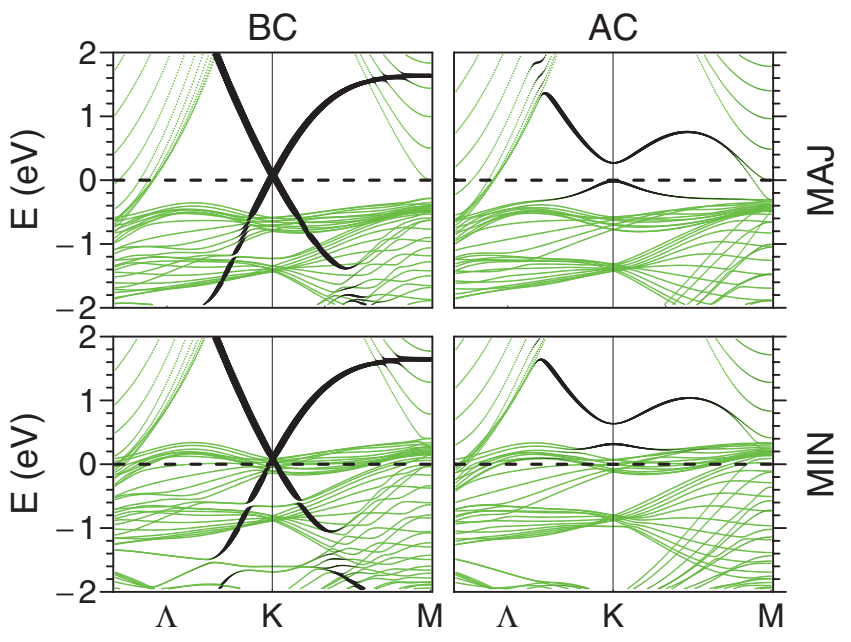

FIG. 4 (color). Majority and minority spin band structures (green) of a single graphene layer absorbed upon (both sides of) a 13 layer (111) Ni slab for a BC configuration with $d=$ $3.3 \AA$, and an AC configuration with $d=2.0 \AA$. The bands replotted in black using the carbon $p_{z}$ character as a weighting factor are superimposed. The Fermi energy is indicated by the horizontal dashed line. 
no graphene states at the $\mathrm{K}$ point in reciprocal space at the Fermi energy for the minority spin channel. This may prevent efficient spin injection into graphene in lateral, current-in-plane devices [32]. However, there is a simple remedy. If a monolayer (or several layers) of $\mathrm{Cu}$ is deposited on $\mathrm{Ni}$, graphene will form only a weak bond with $\mathrm{Cu}$ and the Fermi energy graphene states at the $\mathrm{K}$ point will only be weakly perturbed. $\mathrm{Cu}$ will attenuate the conductance of both spin channels because $\mathrm{Cu}$ has no states at the $\mathrm{K}$ point but will not change the spin-injection properties as long as it is sufficiently ordered as to preserve the translational symmetry; a completely mixed $\mathrm{CuNi}$ top layer reduces the $\mathrm{MR}$ in a $\mathrm{Ni}\left|\mathrm{Gr}_{n}\right| \mathrm{Cu}_{50} \mathrm{Ni}_{50} \mid \mathrm{Ni}$ junction only slightly (Fig. 2). $\mathrm{Cu}$ will also oxidize less readily than the more reactive Ni or Co. Spin-flip scattering in a thin layer of $\mathrm{Cu}$ can be neglected. The weaker bonding of graphene to $\mathrm{Cu}$ may also have practical advantages in sample preparation.

Finally, we remark that graphene may exhibit curious

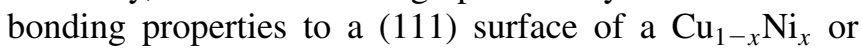
$\mathrm{Cu}_{1-x} \mathrm{Co}_{x}$ alloy; as a function of increasing concentration $x$, the weak minimum at $d \sim 3.2 \AA$ will evolve into a deeper minimum at $d \sim 2.0 \AA$ with the possibility of a double minimum occurring for some range of concentration $x$; a propensity to form a second minimum is already evident in the binding energy curve for $\mathrm{Cu}$. Calculations are under way to examine this possibility.

Planar interfaces between graphene and close-packed $\mathrm{Co}, \mathrm{Ni}$, or $\mathrm{Cu}$ represent a very flexible system for studying the influence of atomic and electronic structure on electrical contact with graphene-related systems such as carbon nanotubes [33] where the nanotube geometry is very difficult to model using materials specific calculations [34]. The binding energy curves in Fig. 3 and electronic structures in Fig. 4 show that the closer proximity resulting from stronger bonding does not necessarily lead to better electrical contact if bonding removes the carbon-related conducting states from the Fermi energy.

Motivated by the recent progress in preparing and manipulating discrete, essentially atomically perfect graphene layers, we have used parameter-free, materials specific electronic structure calculations to explore the bonding and spin transport properties of a novel $\mathrm{TM} \mid \mathrm{Gr}_{n}$ system. We predict perfect spin filtering for ideal $\mathrm{TM}\left|\mathrm{Gr}_{n}\right| \mathrm{TM}$ junctions with $\mathrm{TM}=\mathrm{Co}$ or $\mathrm{Ni}$.

This work is supported by "NanoNed", a nanotechnology programme of the Dutch Ministry of Economic Affairs. It is part of the research programs of "Chemische Wetenschappen" (CW) and "Stichting voor Fundamenteel Onderzoek der Materie" (FOM) and the use of supercomputer facilities was sponsored by the "Stichting Nationale Computer Faciliteiten" (NCF), all financially supported by the "Nederlandse Organisatie voor Wetenschappelijk Onderzoek" (NWO). M.Z. wishes to acknowledge support from EU grant CARDEQ under Contract No. IST-021285-2. This research was supported in part by the National Science Foundation under Grants No. PHY99-07949 (P. J. K.) and PHY05-51164 (J. vdB).

[1] M. N. Baibich et al., Phys. Rev. Lett. 61, 2472 (1988).

[2] G. Binasch et al., Phys. Rev. B 39, 4828 (1989).

[3] S. S. P. Parkin, Applications of Magnetic Nanostructures, Advances in Condensed Matter Sciences Vol. 3 (Taylor \& Francis, New York, 2002), Chap. 5, p. 237.

[4] H. Dery et al., Nature (London) 447, 573 (2007).

[5] B. Trauzettel et al., Nature Phys. 3, 192 (2007).

[6] R. A. de Groot et al., Phys. Rev. Lett. 50, 2024 (1983).

[7] P. A. Dowben and R. Skomski, J. Appl. Phys. 95, 7453 (2004).

[8] G. Schmidt et al., Phys. Rev. B 62, R4790 (2000).

[9] S. Yuasa et al., Nat. Mater. 3, 868 (2004).

[10] S. S. P. Parkin et al., Nat. Mater. 3, 862 (2004).

[11] W. H. Butler et al., Phys. Rev. B 63, 054416 (2001).

[12] J. Mathon and A. Umerski, Phys. Rev. B 63, 220403(R) (2001).

[13] S. Yuasa et al., Appl. Phys. Lett. 89, 042505 (2006).

[14] E. Y. Tsymbal et al., Prog. Mater. Sci. 52, 401 (2007).

[15] M. Zwierzycki et al., Phys. Rev. B 67, 092401 (2003).

[16] P. X. Xu et al., Phys. Rev. B 73, 180402(R) (2006).

[17] H. Ibach and H. Lüth, Solid-State Physics (SpringerVerlag, Berlin, Heidelberg, 1995), 2nd ed.

[18] C. Oshima and A. Nagashima, J. Phys. Condens. Matter 9, 1 (1997).

[19] H. B. Michaelson, J. Appl. Phys. 48, 4729 (1977).

[20] K. Xia et al., Phys. Rev. B 63, 064407 (2001).

[21] K. Xia et al., Phys. Rev. B 73, 064420 (2006).

[22] A. K. Geim and K.S. Novoselov, Nat. Mater. 6, 183 (2007).

[23] D. J. Monsma, R. Vlutters, and J. C. Lodder, Science 281, 407 (1998).

[24] K. Matsubara, K. Sugihara, and T. Tsuzuku, Phys. Rev. B 41, 969 (1990).

[25] P. E. Blöchl, Phys. Rev. B 50, 17953 (1994).

[26] G. Kresse and D. Joubert, Phys. Rev. B 59, 1758 (1999).

[27] G. Kresse and J. Hafner, Phys. Rev. B 47, 558 (1993).

[28] G. Kresse and J. Furthmuller, Phys. Rev. B 54, 11169 (1996).

[29] J. Neugebauer and M. Scheffler, Phys. Rev. B 46, 16067 (1992).

[30] G. Bertoni et al., Phys. Rev. B 71, 075402 (2005).

[31] Y. Gamo et al., Surf. Sci. 374, 61 (1997).

[32] E. W. Hill et al., IEEE Trans. Magn. 42, 2694 (2006).

[33] C. Schönenberger, Semicond. Sci. Technol. 21, S1 (2006).

[34] N. Nemec, D. Tomanek, and G. Cuniberti, Phys. Rev. Lett. 96, 076802 (2006). 